rev.relac.int.estrateg.segur.6(1):191-210,2011

\title{
LA CORTE PENAL INTERNACIONAL ANALIZADA DESDE LA TEORÍA DE LA INTERDEPENDENCIA COMPLEJA
}

Gustavo Sierra Prieto*

\section{RESUMEN}

En la presente investigación se describirá el proceso evolutivo de la Corte Penal Internacional; también se analizará su función a la luz de las características de la Teoría de la Interdependencia en el contexto mundial, teniendo en cuenta canales múltiples de las Relaciones Internacionales, la falta de jerarquización entre los temas a tratar y el menor papel que vienen desempeñando las Fuerzas Militares en la solución de los problemas internacionales. Finalmente, concluir que la cooperación judicial es esencial para evitar la impunidad en los delitos de lesa humanidad.

Palabras clave: múltiples actores, delitos de lesa humanidad, genocidio, crimen de guerra, actores dominantes, actores no estatales e interdependencia.

* Magister en Relaciones Internacionales. Correo electrónico: gusipri@yahoo.com 


\begin{abstract}
The present study will describe the evolutionary process of the International Criminal Court, and will also assess its role in light of the characteristics of the Theory of Interdependence in the global context, taking into account multiple channels of International Relations, the lack of ranking among the topics to be dealt and the diminished role that the Armed Forces have been playing in resolving international problems. Finally, it concludes that judicial cooperation is essential to avoid impunity in crimes against humanity.
\end{abstract}

Keywords: multiple actors, crimes against humanity, genocide, war crime, dominant actors, non-state actors, interdependence

\title{
RESUMO
}

Esta pesquisa irá descrever o processo da evolução do Tribunal Penal Internacional e também analisará o seu papel em relação às características da Teoria da Interdependência no contexto global, levando em consideração os vários canais das Relações Internacionais, a falta de hierarquia entre os tópicos a serem abordados e o menor papel que vêm desempenhando as Forças Militares na solução dos problemas internacionais. Para finalmente concluir que a cooperação judicial é essencial para evitar a impunidade nos crimes contra a humanidade.

Palavras chave: múltiplos atores, crimes contra a humanidade, genocídio, crimes de guerra, atores dominantes, atores não estatais e interdependência.

\section{INTRODUCCIÓN}

Los delitos de Lesa Humanidad que a través de la historia se han venido presentando en las diferentes regiones del mundo han sido tema de preocupación de la Organización de las Naciones Unidas ONU; por esta razón, después de la Segunda Guerra Mundial, y en la década de los noventa, se crearon los tribunales militares de Nuremberg, Tokio, Ruanda y Yugoslavia como herramientas jurídicas y mecanismos temporales de la comunidad internacional para proteger la violación de los Derechos Humanos y, en especial, del Derecho Internacional Humanitario.

Es así como el 17 de julio de 1998, en reunión de plenipotenciarios de la Asamblea General de las Naciones Unidas se aprueba el Estatuto de Roma, instrumento que crea la Corte Penal Internacional a fin de que esta institución quede facultada para ejercer su jurisdicción sobre personas respecto de los crímenes más graves de trascendencia internacional y además funcione con carácter complementario para las jurisdicciones penales nacionales. 
En el presente artículo se va desarrollar en primera instancia el proceso evolutivo por el cual se crea la Corte Penal Internacional, como un nuevo actor internacional que busca juzgar a todos aquellos individuos que violen los Derechos Humanos y el Derecho Internacional Humanitario.

En segunda instancia se va realizar un análisis detallado del funcionamiento de la Corte Penal Internacional en el actual contexto mundial, teniendo como base la Teoría de la Interdependencia Compleja, pues aunque la aplicación de la Corte genera vulnerabilidad para la soberanía de los Estados, la Teoría antes mencionada permite reconocer que cualquier organización que desempeñe un papel importante jurídicamente en el sistema internacional, se convierte en un actor que contribuye al equilibrio mundial. Por tal razón, la Corte Penal Internacional pretende con su aplicación cubrir esa deficiencia jurídica de los Estados para castigar delitos de lesa humanidad dentro de su soberanía y de esta forma promover y mantener un orden en el sistema internacional.

Así mismo, se analiza la función de esta Institución a la luz de las características de dicha teoría y cómo estas pueden explicar el funcionamiento de la Corte Penal Internacional en el contexto mundial, teniendo en cuenta los canales múltiples de las Relaciones Internacionales y la falta de jerarquización entre los temas a tratar y el menor papel que vienen desempeñando las Fuerzas Militares en la solución de los problemas internacionales, debido al realce jurídico en la solución de problemas concernientes a la protección de los Derechos Humanos y el Derecho Internacional Humanitario.

Lo anterior, a fin de percibir la evolución de la CPI en el contexto mundial y la necesidad que tienen los Estados en un momento dado de la cooperación judicial para evitar la impunidad en los delitos de lesa humanidad.

El desarrollo evolutivo de las relaciones internacionales ha generado a través de la historia una serie de teorías explicativas que intentan dar claridad conceptual a acontecimientos concernientes en materia de política internacional.

Estas teorías surgen de analistas internacionales de diferentes regiones del mundo, los cuales, acordes con el proceso evolutivo de la comunidad internacional, pretenden ajustar las nuevas herramientas e instrumentos de la política internacional a las realidades actuales, a fin de fijar las relaciones entre los nuevos actores internacionales.

Lo anterior permite entender y analizar de forma apropiada la existencia y evolución de entidades o cuerpos jurídicos que en el ámbito transnacional han logrado generar una transformación básica en los patrones de interacción de los diferentes actores internacionales y en la reacomodación de las dinámicas internas de estos, principalmente si se habla de los Estados.

Bajo este concepto, es de analizar el nuevo rol que la Corte Penal Internacional va a jugar en la comunidad internacional basado en la Teoría de la Interdependencia Compleja, la cual deja de 
lado el sentido realista de las relaciones de poder que define a los Estados como los únicos organismos capaces de generar relaciones internacionales e involucra al desarrollo de estas relaciones organismos e individuos que de carácter transnacionales afectan las relaciones políticas, sociales y culturales de los Estados.

El desarrollo de la Teoría de la Interdependencia que se ha fijado bajo un modelo idealista, nos permite acercarnos a la eficacia y el rol que entraría a desempeñar la Corte Penal Internacional en el contexto mundial.

La Teoría de la Interdependencia afirma que "podemos imaginarnos un mundo en el que hay actores no-estatales que participan activamente en la política mundial, en el que no existe una jerarquía clara entre los diversos tipos de asuntos, y en el que la fuerza no es efectiva como instrumento de la política exterior" (R.O. Keohanne \& J.S. Nye., 1977).

De esta definición podemos extraer los tres componentes principales de la Teoría de la Interdependencia Compleja. En primer lugar, la existencia de actores no-estatales que participan de la política mundial generando una multiplicidad de canales para encaminar las relaciones internacionales e interactuar en las políticas internacionales. Canales como las relaciones interestatales y transnacionales, e incluso transgubernamentales, se ven reflejados en mecanismos que se han consolidado a través de los años como lo son los tratados de libre comercio, organismos internacionales como la Unión Interparlamentaria, que es una institución en la cual intervienen los parlamentos de diferentes Estados; la existencia de Organizaciones No Gubernamentales como participantes activos de situaciones coyunturales y de conflictividad y violación de los Derechos Humanos y el Derecho Internacional Humanitario dan muestra de que estos canales múltiples han desempeñado un papel satisfactorio en las actuales relaciones de poder. La Corte Penal Internacional es uno de estos canales en las actuales relaciones políticas, sociales y culturales en el contexto internacional.

En segundo lugar, la ausencia de una jerarquización clara de los diferentes asuntos que rigen las relaciones de los Estados permite que aspectos como la economía y el poder militar se impongan a factores de tipo social, político o de desarrollo de los Estados. La Teoría de la Interdependencia bajo este criterio busca una equidad en el manejo de las relaciones de los Estados a nivel internacional, donde no se persigan intereses específicos por parte de las potencias y se genere un aprovechamiento de condiciones frente a los Estados en vías de desarrollo, sino por el contrario se mantengan en equilibrio las relaciones internacionales, evitando la corrupción o el malestar en cualquiera de sus estamentos. La Corte Penal Internacional es un organismo que persigue este principio, pues no busca discriminar ninguna situación en alguna región del mundo, por razones políticas, económicas y sociales, sino que, por el contrario, pretende bajo unos altos niveles de imparcialidad y objetividad juzgar a los individuos en los diferentes países del mundo siempre que se violen los Derechos Humanos y el Derecho Internacional Humanitario. 
Uno de los antecedentes de la falta de aceptación de este principio por parte de las potencias es el hecho de que Estados Unidos, como primera potencia internacional, no haya ratificado el Estatuto de Roma; este hecho corrobora las sospechas de que la interdependencia compleja afecta en gran medida los intereses de algunas potencias ya consolidadas.

Por último, encuentro que el poder militar no es una prioridad dentro de la Teoría de la Interdependencia. La política exterior de los actores del sistema internacionales, con el pasar del tiempo y con el fin de la carrera armamentista entre Estados Unidos y la Unión Soviética, abrieron paso a la diplomacia y a la negociación como opciones para fomentar las relaciones internacionales. El uso de la fuerza debe ser limitado en el fomento de relaciones con otros Estados y regiones.

La Corte Penal Internacional y su funcionamiento en el contexto internacional se identifican claramente dentro de esta Teoría de la Interdependencia Compleja, que en últimas reduce la autonomía de las grandes potencias para el manejo de asuntos como la paz y la seguridad internacional y busca a través de sus mecanismos jurídicos encontrar un equilibrio para proteger los Derechos Humanos y el Derecho Internacional Humanitario en los diferentes escenarios del mundo.

\section{LA CORTE PENAL INTERNACIONAL}

\section{Antecedentes}

Para entender lo que es hoy la Corte Penal Internacional se tiene que hacer referencia a hechos históricos, que son la base de la creación de esta institución. Uno de los primeros juicios internacionales que se conoce aconteció en el siglo IV cuando un noble francés llamado Jean de Melun fue rehén de un inglés de nombre Poinfroit, los dos llegaron a un acuerdo para que Melun recobrara su libertad. Él debía abonar una cantidad determinada la cual fue pagada de inmediato, sin embargo, el noble tendría que pagar lo restante cuando estuviera en libertad; para que esto se cumpliera, se dejó constancia en un escrito en el cual Melun se comprometía a pagar lo acordado; si esto no se llevaba a cabo, perdería de nuevo su libertad.

Melun al recobrar su libertad no quiso pagar y, debido a esto, el caso se llevó a un arbitraje donde se pudiera dirimir, este falló a favor del francés; sin embargo, Henri Poinfroit llevó su caso a la Corte de los Mariscales, en donde determinaron que el noble tenía la obligación de acatar lo que se había acordado, pero este no aceptó y lo presentó en el Parlamento de París donde lo respaldaron, pues el documento no tenía los requisitos necesarios y aceptados por el rey, para que este se hiciera efectivo.

El noble francés aceptó la legitimidad del documento, pero aclaró que se dio por presiones e intimidaciones y demostró que al ser inválido el documento, él no tenía por qué saldar el dinero 
de la liberación, además exigió que el inglés le devolviera el dinero que pagó por su rescate. "Al darse cuenta de que podía obtener la nulidad de su promesa, abandonó su argumento inicial y ahora exigía que además del reembolso del dinero se le pagaran los daños por los insultos gratuitos constituidos por el hecho". (CÓRDOBA TRIVIÑO, JAIME. "Derecho Penal Internacional". 2001).

El Tribunal determinó que el documento era nulo y que el inglés estaba en la obligación de devolver el dinero que le quitó a Melun y además de esto debía pagarle por los daños causados, y hasta que pagara estaría en prisión.

En esta época se dieron varios hechos como este, pues los hombres al estar en estas contiendas se supeditaban a unas normas que se hacían cumplir por medio de cortes y tribunales, las cuales se crearon para enjuiciar a los hombres que no cumplieran con las normas establecidas para los momentos de guerra, sin importar el país de origen de estos.

Un ejemplo claro que se resalta como precedente en los juicios internacionales se dio en el siglo V cuando se enjuició a un hombre llamado Peter Von Hagenbach por las barbaries perpetradas a la población de Breisach, la cual quería subyugar ante la ciudad de Borgoña (Francia). Hagenbach fue enjuiciado por una corte integrada por jueces de diferentes lugares del Continente europeo.

Hagenbach era la autoridad en Breisach y seguía las disposiciones del Duque de Borgoña. Von Hagenbach estableció un régimen de terror para someter a la población y gobernarla. "Los hechos realizados por el gobernador consistían en homicidios, violaciones, imposición de gravámenes y confiscaciones arbitrarias" (CÓRDOBA TRIVIÑO, JAIME. "Derecho Penal Internacional". 2001).

Esta situación se tornó peligrosa para los habitantes de esta provincia, como también para los países que estaban en la frontera; por esto se organizó un plan para arrestar a Hagenbach, lo cual se logró. "Se estableció una corte ad-hoc conformada por 28 jueces de la coalición y un presidente designado por el Archiduque de Austria, en su calidad de regente de los destinos de los habitantes de Breisach". (CÓRDOBA TRIVIÑO, JAIME. "Derecho Penal Internacional". 2001).

Los casos anteriores son un ejemplo de cómo en esta época se trató de dar soluciones a situaciones que no estaban en manos de los Estados, sino más bien fuera de su alcance, y para darle una solución a estos acudieron a otros procedimientos. Pero estas experiencias que se dieron en la Edad Media no pueden tomarse como el surgimiento de la Corte Penal Internacional; esto ha sido un proceso, pues en un principio se juzgaban pocos delitos los cuales tenían que ver solo con hechos donde se infringían las conductas establecidas y las leyes para los enfrentamientos bélicos, hoy en día los tribunales van más allá, debido a que no solo juzgan crímenes que se cometen en la guerra, sino también otros que tienen que ver con todo lo concerniente a la protección de los Derechos Humanos de los ciudadanos. 
Es importante resaltar los aportes que se dieron en la Guerra Civil de Estados Unidos, en la cual se establecieron los actos que se pueden dar en un conflicto, como son los crímenes de guerra donde se compilaron algunas normas (Código de Lieber), las cuales hacían referencia a diferentes situaciones que se presentaban en la guerra. "Así mismo, se castiga la destrucción de la propiedad y las conductas de robo, pillaje o saqueo, la violación, las lesiones personales y la mutilación o el asesinato de las personas que no toman parte en el conflicto, es decir, lo que hoy se entiende como población civil no combatiente". (Greppi, E., 1999).

Este Código fue el antecesor de todas las leyes que se dieron posteriormente para las situaciones de guerra y la normativa internacional humanitaria; Con esto se trató de humanizar la guerra.

Otro de los avances para la creación de la Corte Pena Internacional fue el Tratado de Versalles, donde se estableció la responsabilidad internacional y en el cual se formula el crimen de agresión, el cual vulnera la estabilidad internacional; pero debido a la falta de cooperación de los Estados, estos tribunales no funcionaron como se esperaba, pues algunos países se negaron a extraditar a los hombres que estaban involucrados en estos actos.

Sin embargo, el Tratado de Versalles sentó un precedente para la creación de los tribunales militares de Tokio y Nuremberg, aunque estos fallaron en algunos aspectos debido a que se dio una confusión entre los crímenes de guerra y los de lesa humanidad, entre otros; además, fueron pocas las personas que condenaron por estos hechos, debido a que había presiones políticas, como por ejemplo, que no juzgaron a algunos fascistas en Italia, pues ellos podrían ayudar a la contención del comunismo en Europa y esto beneficiaría a algunos países vencedores que eran capitalistas; sin embargo, "La existencia de estos tribunales sirvió de mucho para el desarrollo de la responsabilidad penal internacional". (Córdoba , J., 2001).

De todas maneras se juzgaron a unos individuos y se condenaron y, por lo tanto, los hechos materia de investigación no quedaron impunes.

Estas experiencias mostraron la falta de normatividad para definir los crímenes que se cometieron en la Segunda Guerra Mundial, pues la humanidad nunca había vivido este tipo de enfrentamientos y situaciones; debido a esto se incurrió en la violación del principio de legalidad, debido a que no había leyes ni códigos que establecieran cuáles eran los delitos por los cuales se debía condenar a una persona. "Con este principio, finalmente, se busca establecer una clara distinción entre el bien y el mal y entre la moral y el derecho, pues con la ley penal se señala aquello que el Estado considera lesivo para los intereses de la sociedad o para sus propios intereses", es decir, se tipifican claramente las conductas delictivas en la norma.

Después de la Segunda Guerra Mundial, la Organización de las Naciones Unidas consideró que era importante crear un código de crímenes de guerra de la humanidad que incluyera los 
crímenes de lesa humanidad, genocidio, crimen de guerra y agresión, para que una Corte Penal Internacional juzgara a las personas que cometieran estos delitos, pero por diferencias en algunos puntos, como el del crimen de agresión, no se logró definir y por esto su desarrollo se vio suspendido por algunas décadas. "Pese a estos esfuerzos, durante la guerra fría y hasta la década del noventa no hubo preocupación alguna por parte de la comunidad internacional para tipificar este tipo de crímenes". (Córdoba, J., 2001).

A principios de los noventa, debido a los enfrentamientos que se estaban presentando en la antigua Yugoslavia, diferentes grupos presionaron a la Organización de las Naciones Unidas para que mediara; se decidió realizar una investigación por parte de la ONU para saber qué estaba sucediendo en ese país; aunque hubo muchos tropiezos para recoger los datos e informes sobre el conflicto, el Consejo de Seguridad de las Naciones Unidas, al enterarse de lo que estaba sucediendo, creó un Tribunal Internacional Ad-hoc para la antigua Yugoslavia.

"El tribunal se ocupa del juzgamiento de las serias violaciones al Derecho Internacional Humanitario cometidas en el territorio de la antigua Yugoslavia desde 1991. Los crímenes sometidos a su competencia son los siguientes: 1) Las violaciones a las leyes y costumbres de la guerra, 2) los graves incumplimientos de las Convenciones de Ginebra de 1949, 3) el genocidio, y 4) los crímenes contra la humanidad. La competencia del tribunal se ejerce sobre todas las personas que hayan cometido estos crímenes en el territorio de la antigua Yugoslavia desde 1991, sin importar el bando al cual pertenezca el responsable". (Córdoba, J., 2001).

Así mismo, en 1994 se creó una comisión que se encargó de investigar la vulneración de los derechos humanos en Ruanda, en los enfrentamientos que se estaban dando entre los Humus y Tutsis; aunque a esta comisión le dieron pocos meses para indagar sobre estos hechos, la Organización de las Naciones Unidas recibió un comunicado de la situación y decidió crear un tribunal Ad-hoc para Ruanda. "Este tribunal se caracteriza porque su competencia recae sobre actos cometidos en un conflicto armado de carácter no internacional, en tanto que el Tribunal Ad-hoc para la antigua Yugoslavia se ocupa de actos cometidos en un conflicto armado de carácter internacional, aunque en sus orígenes este fue uno de tipo no internacional". (HARHOFF, FREDERICK. Revista Internacional de la Cruz Roja, 1997).

Para llegar a una Corte Penal Internacional de carácter permanente y con competencia universal, se pasó por los hechos históricos anteriormente mencionados; la idea de crear este Tribunal se retomó en 1990 tomando como base algunos convenios y tribunales que se dieron en el siglo XX.

En la sesión 52 del 17 de julio de 1998, la Asamblea General adopta el "Estatuto de Roma de la Corte Penal Internacional, por 120 votos a favor y 21 abstenciones de países como los Estados Unidos, China, India, Israel, Sudán, Libia y Qatar". (Córdoba, J., 2001). 


\section{CARACTERÍSTICAS DE LA TEORÍA DE LA INTERDEPENDENCIA_COMPLEJA}

La Interdependencia Compleja da origen a diferentes procesos políticos. En algunos sentidos el modelo de la Interdependencia Compleja nos muestra la seguridad donde el escenario es global, formulando que se acerca más a la realidad que el realismo y partiendo de las siguientes premisas centrales:

Los Estados, tratados metodológicamente como si fueran unidades coherentes, son los actores dominantes de la política mundial; la fuerza es un instrumento usable y efectivo de la política exterior, pero las relaciones entre los Estados según la Interdependencia Compleja debe basarse en la cooperación e intercambio de todas las fuerzas vivas del Estado, desde el punto de vista gubernamental y no gubernamental y no desde la fuerza.

Existe una jerarquía de ámbitos o asuntos en la política mundial, encabezada por la seguridad militar: la alta política de la seguridad militar domina a la baja de los asuntos económicos y sociales, según la teoría del realismo de Hans Morguentau.

Cada una de estas premisas puede ser desafiada según la Teoría de la Interdependencia Compleja y "podemos imaginarnos un mundo en el que hay actores no-estatales que participan activamente en la política mundial, en el que no existe una jerarquía clara entre los diversos tipos de asuntos, y en el que la fuerza no es efectiva como instrumento de la política (exterior)". (Keohane, R. \& Nye, J., 1998).

La Interdependencia posee tres características primordiales que le dan una base sólida a las relaciones intangibles entre los Estados, y son las siguientes:

\section{LOS CANALES MÚLTIPLES}

Entre los canales múltiples se pueden recapitular básicamente "las relaciones interestatales, transgubernamentales y transnacionales" (KEOHANE, ROBERT O., NYE, JOSEPH S. "Poder e Interdependencia". 1998), que son concebidas por los realistas partiendo de que los Estados son unidades y actores que adhieren las sociedades, es decir, que de un modo u otro las élites existentes tales como las empresas, los gobiernos de cada país, las multinacionales, entre otras, son grupos influyentes en las políticas tanto internas como externas, pues realizan actividades lícitamente establecidas que interfieren como conectivos cada vez más entre los países.

Dichas conexiones entre estos canales dan paso a que las dificultades políticas exteriores vayan en ascenso; esto se debe a que muchas de las decisiones tomadas entre cada uno de los canales choquen entre los mismos y, por lo tanto, no se puede mantener una comunicación sólida en donde los intereses no sean conjuntos sino individuales. 
Por otro lado, esta característica de la Interdependencia Compleja puede amortiguar los inconvenientes, las dificultades y los problemas que se puedan presentar en estos nexos comunicativos entre los actores, pues ofrece una flexibilidad de los procesos explícitos y una integridad en la coherencia de los Estados como unidad.

Como reflexión a lo anterior, es preciso decir que donde hay gran diferencia en las relaciones de las firmas privadas o empresas privadas es donde hay una sociedad cerrada pensando solo en su propio bien, no dejando atrás que el gobierno tiene un rol especial en sus dinámicas y organizaciones, donde las políticas económicas entrelazan las fronteras internas y externas resaltando la cantidad de problemas relevantes para la política exterior en su fondo, sea gubernamental o no gubernamental.

\section{FALTA DE JERARQUIZACIÓN ENTRE LAS CUESTIONES}

"Las agendas de asuntos exteriores, es decir, conjuntos de cuestiones relevantes para la política exterior en los que se hallan involucrados los gobiernos, se han tornado más amplias y más diversas. Ya no pueden subordinarse todos los temas al de la seguridad militar". (KEOHANE, ROBERT O., NYE, JOSEPH S. "Poder e Interdependencia". 1998).

Existen muchos temas de la agenda externa e interna que no abarcan todas las necesidades, es decir, que soportan las ayudas dirigidas solo a lo militar y olvidan temas como los problemas energéticos, la emergencia ambiental y social y los conflictos internos.

Además, la falta de una real agenda diplomática conlleva a una rivalidad territorial, económica, social, política y cultural que da como resultado una inestabilidad dentro de los países.

Con respecto a lo anterior, se demuestra que no se observa un verdadero progreso de los Estados en todas sus generalidades (agenda política interna y externa, económica y cultural, entre otras), porque se ve reflejada la desigualdad entre los países desarrollados y en vía de desarrollo (brecha entre países pobres y ricos), que cada vez se va viendo más grande y sus posibles soluciones son más escasas.

\section{MENOR PAPEL DE LAS FUERZAS MILITARES}

"La fuerza militar no es empleada por los gobiernos contra otros gobiernos de la región cuando predomina la Interdependencia Compleja". (KEOHANE, ROBERT O., NYE, JOSEPH S. "Poder e Interdependencia". 1998).

Por tanto, es de gran importancia para los gobiernos este punto de la agenda, porque dentro de los gobiernos de la región debe predominar la Interdependencia Compleja, debido a que las 
fuerzas militares pueden ser la opción menos viable para resolver desacuerdos entre las partes y, por ende, los aspectos económicos, sociales y culturales pueden ser fundamentales para dirimir las diferencias, no solo con entes gubernamentales, sino también con entes no gubernamentales.

\section{LA CORTE PENAL INTERNACIONAL Y LA TEORÍA DE LA INTERDEPENDENCIA COMPLEJA}

La Teoría de la Interdependencia Compleja se caracteriza, de acuerdo con lo planteado en Poder e Interdependencia, (KEOHANE, ROBERT O., NYE, JOSEPH S. 1998), por tres rasgos principales: en primer lugar, la existencia de múltiples actores además de los Estados que participan activamente en la política mundial; en segundo lugar, la inexistencia de una clara jerarquía de cuestiones y, por último, la fuerza como un instrumento de política ineficaz. Sin embargo, vale la pena aclarar que en el desarrollo de este punto no pretendo darle una explicación "teoricista" a la Corte Penal Internacional ni mucho menos explicar la compleja realidad en la que se inscribe, sino simplemente realizar una confrontación entre un discurso y un escenario específico, como lo puede ser la aplicabilidad de los Derechos Humanos y el Derecho Internacional Humanitario a través de la gestión de una institución jurídica y consensuada internacionalmente.

Por esta razón se debe tener en cuenta cada una de las características anteriormente enunciadas de la Interdependencia Compleja y explicarlas de acuerdo con este tribunal internacional.

\section{CANALES MÚLTIPLES}

Es preciso analizar, en primer lugar, la amplitud de las relaciones que se gestan entre las élites gubernamentales y las organizaciones transnacionales; de tal forma tales relaciones se identifican en tres niveles diferentes: interestatales, transgubernamentales y transnacionales.

En el caso de las relaciones interestatales, la interrelación entre los actores es algo rescatable de la escuela realista, pues estas se consolidaron como instrumento de poder estratégico de los Estados para gestar los beneficios o resultados esperados con base en el poder militar que dichas entidades políticas poseían en proporción a las amenazas a su seguridad nacional. Por tanto, en este sentido ese relacionamiento se evidenció, de una parte, en las dinámicas que precedieron la creación de la Corte Penal Internacional, debido a que algunos Estados mantuvieron un alto grado de comunicación directa entre sí, por la búsqueda de consensos sobre determinadas cuestiones tales como la aplicabilidad del Estatuto de Roma en el ámbito interno de los Estados, la legislación aplicable o la tipificación de los crímenes a juzgar; y de otra parte, en lo que se refiere al compromiso unilateral de cada uno de los Estados con la ratificación y entrada en vigor del Estatuto; todo lo cual se vio influenciado por las constantes negociaciones bilaterales que 
Estados Unidos a nombre propio adelantó con el objeto de ponderar la capacidad jurídica de la Corte sobre el estatus de sus ciudadanos.

De tal forma, al hablar de las relaciones transgubernamentales ${ }^{1}$ es posible afirmar que la gestación de coaliciones y todo tipo de alianzas entre los gobiernos, con el objeto de procurar un apoyo a la materialización del derecho penal internacional a través de la cooperación intergubernamental necesaria, le ha permitido a la Corte establecerse como una instancia jurídica internacional de primer orden para los objetivos humanitarios de aquellos Estados profundamente interesados y comprometidos con la reivindicación de los derechos fundamentales de la sociedad civil mundial, lo cual es una preocupación creciente si se analiza la multiplicidad de amenazas (terrorismo, violencia organizada, narcotráfico, pobreza, discriminación, entre otras) que durante los últimos años han llegado a posicionarse en los primeros lugares de las agendas políticas de diversos Estados.

En cuanto a las relaciones transnacionales ${ }^{2}$, es fundamental analizar cómo con la institucionalización de entes como Naciones Unidas, a través de sus agencias especializadas y el surgimiento de organizaciones de orden civil internacional tales como las Organizaciones No Gubernamentales o los movimientos sociales de orden interestatal (cuyos objetivos de reivindicación de derechos laborales, de género, étnicos o culturales los consolida como una fuerza importante), el fin y el objeto de la Corte se ve reafirmado, debido principalmente a la doble función de fiscalización y recolección de información que estas organizaciones junto con los Estados desempañan, lo que configura una compleja red de relaciones transnacionales que en mucho contribuye a la democratización de la gestión de las relaciones internacionales, tal como lo anotan Keohane y Nye al afirmar que "...estos actores son importantes no solo por sus actividades en la concreción de sus propios intereses, sino también porque actúan como correas de transmisión haciendo más sensibles a las políticas gubernamentales en distintos países...".

Todo lo anterior permite reconocer la importancia del Estado en la configuración de todas estas relaciones y nos remite a las preguntas de si zel Estado se ha visto disminuido en su papel de principal gestor de las interacciones a nivel internacional? y, por consiguiente, si zel principio de soberanía que arguyen algunos especialistas se ve trasgredido por la existencia de organismos tales como la Corte Penal Internacional? Para responder a estos cuestionamientos es preciso evaluar el peso de las decisiones de los Estados frente a la posibilidad de intervención de la Corte en la función jurisdiccional de estos, lo cual constituye uno de los retos que las naciones deben afrontar de la misma manera como han logrado sobrellevar los rigores de la mundialización de

1. Keohane, R., Nye, J. "Las relaciones transgubernamentales aparecen cuando se flexibiliza el supuesto realista de que los Estados actúan coherentemente como unidades". Poder e Interdependencia. p. 41.

2. Keohane, R., NYE, J. "Las relaciones transnacionales surgen cuando se flexibiliza el supuesto de que los Estados son las únicas unidades". Poder e Interdependencia. p. 41. 
las economías y el aumento del intercambio comercial internacional; así es preciso que los gobiernos se comprometan a asumir la función de velar y garantizar el buen desempeño del poder judicial frente a los casos que sobre crímenes de lesa humanidad, crímenes de guerra, genocidio y agresión que se le presentan y de esta forma reafirmar el "ejercicio efectivo de poder sobre un territorio determinado", tal como lo prescribe el conocido concepto de soberanía.

\section{FALTA DE JERARQUIZACIÓN ENTRE LAS CUESTIONES}

Con la complejización de las relaciones internacionales en el marco de la Interdependencia es entendible la dificultad que representa la construcción de la agenda de asuntos exteriores a nivel estatal y mundial, lo cual de una forma tácita determina al tipo de relaciones y las acciones que a nivel internacional los diversos actores luchan por forjar.

Recientemente, el desarrollo de nuevas problemáticas y fenómenos a nivel nacional, regional y global ha permitido una reflexión más amplia sobre la importancia de incluir dichas temáticas en las agendas y de esta forma lograr cierto nivel de "politización" de los asuntos que en otro contexto tendrían un tratamiento diferente. Este es el caso de los temas referentes a los Derechos Humanos y al Derecho Internacional Humanitario, pues hasta tiempos recientes la Agenda Global estaba determinada por las directrices de las políticas militares y de seguridad nacional, lo cual determinó que en las agendas internas de los Estados se asumiera una clara filiación con estas premisas y así se llegara a ajustar todo interés económico, social o cultural al paradigma político de los "intereses nacionales".

En tanto la Corte Penal Internacional representa una pretensión del Sistema Internacional por reorientar los esfuerzos de los actores hacia los temas que afectan directamente la dignidad y la naturaleza humana, parece ser incomprensible para los agentes violadores del Derecho Internacional Humanitario el hecho de la existencia jurídica y política de dicha instancia y, por lo tanto, estos tratan de ignorarla o evadirla amparándose o acogiéndose a los procesos y garantías que los Estados ofrecen. Esto se debe en gran medida a que para los Estados, específicamente y en un contexto interdependiente como el actual, les es necesario y fundamental mantener niveles de consistencia y coherencia entre su política interna y externa, situación que es aprovechada por grupos o individuos (comprometidos en actos punibles) para propiciar el repliegue y aislamiento del Estado de dichas dinámicas internacionales; por tanto, tal como lo exponen Keohane y Nye: "Las ocasiones para la demora, para la protección especial, para la inconsistencia y para la incoherencia abundan cuando las políticas internacionales requieren el alineamiento de las políticas internas de los países democráticos y pluralistas". (KEOHANE, ROBERT O., NYE, JOSEPH S. "Poder e Interdependencia". 1998).

En este sentido es fundamental entender que "...en ausencia de una clara jerarquización de los problemas, las metas variarán según las cuestiones y pueden no estar estrechamente 
relacionadas con ellas. Cada burocracia se mueve en pos de sus propios intereses y aunque varios organismos puedan llegar a compromisos sobre cuestiones que afecten a todos, hallarán que es difícil mantener un patrón político consistente". Lo anteriormente citado nos ubica frente a dos cuestiones básicas en el contexto de la Corte Penal Internacional; de un lado, la conciliación entre los intereses fundamentales de los actores y las cuestiones humanitarias que intentan posicionarse en los ámbitos interno y externo; y de otro, la capacidad real de los Estados o instituciones para mantener y reforzar políticas a largo plazo que proporcionen seguridad y continuidad a los compromisos internacionales asumidos por estos.

Con relación al primer aspecto, se podrían percibir todavía ciertos rezagos del realismo, pues si bien las condiciones o actores del contexto internacional han cambiado, aún los Estados, junto con otros entes como las organizaciones internacionales, invocan y anteponen sus "intereses nacionales" (en el caso de los Estados) o "intereses organizacionales" (en el caso de las organizaciones internacionales) a la adopción de decisiones en materia de política exterior y más aún si se trata, en particular, de temas sobre DIH debido al "relativismo" que se pretende imponer al observar las complejas situaciones de violencia y confrontación armada a nivel mundial; así se explica en Poder e Interdependencia cuando se plantea que "...el Estado debe probar ser multifacético y aun esquizofrénico. Los intereses nacionales habrán de ser definidos en forma diferente ante problemas diferentes, en ocasiones diferentes y por diferentes unidades gubernamentales..." (KEOHANE, ROBERT O., NYE, JOSEPH S. 1998).

De cierta forma esta situación recrea las características de un modelo interdependiente como el imperante, pues de una parte se deben estimar las relaciones de poder condicionantes de dichos "intereses nacionales u organizacionales" presentes en los actuales esquemas de actuación internacional $y$, de otra, se debe tener en cuenta la existencia de instituciones supranacionales y la realidad de su influencia en las esferas domésticas de los Estados y organizaciones.

El segundo aspecto es un problema al interior de los Estados, principalmente, debido a que dadas las dinámicas democráticas de rotación del poder a través de la elección popular, la implementación de políticas de gobierno es un síntoma generalizado que en poco o en nada contribuye al refuerzo de procesos a largo plazo y que, por lo tanto, en el plano externo se constituye en un detractor de la confianza y credibilidad que un Estado debe procurar mantener en sus relaciones de interdependencia ${ }^{3}$; y sobremanera si se trata de compromisos en materia de derecho internacional tal como sucede con los países que ratificaron el Estatuto de Roma, pero que debido a un cambio en las perspectivas de gobierno han retrasado la entrada en vigor de la Corte.

3. Keohane, R., Nye, J. "Los Estados que se hallen mejor colocados para mantener su coherencia (a causa de una tradición política centralizada, como Francia) estarán en mejores condiciones de manipular la interdependencia desigual que los Estados fragmentados, los que a primera vista parecen tener más recursos en un área de cuestiones". Poder e Interdependencia. Buenos Aires: Grupo Editor Latinoamericano. 1998, p.53. 
De otra parte es imprescindible analizar cómo el ejercicio de justicia de la Corte Penal Internacional contribuye al proceso de jerarquización de temas en la Agenda Global, debido a que este órgano reacciona constantemente a las crecientes amenazas a la dignidad humana mundial y, por tanto, permite vislumbrar una reorganización de responsabilidades y redistribución de poder en torno a dichas prioridades. Además, por su carácter multilateral, la Corte trata de incluir una diversidad de perspectivas que posibilita día a día una dialéctica en cuanto a conceptos, mecanismos y políticas que, en definitiva, contribuyen al pluralismo del Sistema Internacional y a la descentralización del proceso de toma de decisiones de los centros de poder tradicionales.

\section{MENOR PAPEL DE LA FUERZA MILITAR}

Un punto clave de la Interdependencia es la pérdida de preponderancia de la fuerza militar como principal herramienta en las relaciones internacionales; por tanto, la diversificación de actores en el escenario internacional ha contribuido a tal fin, por cuanto entes diferentes a los Estados (ONG's, organizaciones internacionales, movimientos sociales, etc.) han logrado consolidar cuotas de poder considerables sin llegar a recurrir al fortalecimiento bélico como bandera de sus acciones ${ }^{4}$.

Este presupuesto es importante si se pretende analizar el relacionamiento entre los llamados Estados "fuertes" y Estados "débiles" que, en definitiva, en el contexto de interdependencia imperante ha conseguido reajustarse en términos de las posibilidades que tiene cada una de estas entidades con el objeto de consolidar sus intereses. De tal forma, es posible observar cómo en el caso de los Estados considerados "poderosos" les ha sido imprescindible replantear el factor militar como principal argumento en asuntos de índole económica, ambiental o social, pues si bien el poderío militar sigue siendo un factor de disuasión importante, no logra consolidarse como determinante en un contexto de redes interdependientes que posibilitan las alianzas como respuestas concertadas a estas amenazas.

La actuación de los Estados denominados "oprimidos" ha tomado preponderancia, gracias al desarrollo de los organismos internacionales, pues ante la existencia de canales múltiples de interacción era muy factible la consolidación de entes supranacionales que, partiendo del concepto de cooperación multilateral, lograran concertar acuerdos, tratados, convenciones o decisiones que de alguna forma aportaran a la solución de problemáticas que no solo afectaban a determinadas poblaciones, sino que amenazaban con perjudicar irreversiblemente a toda la humanidad. Este fue el caso de los foros ambientales y de derechos humanos, y dentro de estos

4. Keohane, R., Nye, J. "El insignificante papel de la fuerza nos lleva a esperar que los Estados dependan más de otros instrumentos a los efectos de ejercer el poder (...) los Estados menos vulnerables tratarán de emplear la interdependencia asimétrica en grupos particulares de cuestiones como una fuente de poder; también tratarán de usar las organizaciones internacionales, los actores transnacionales y los flujos de dinero". Poder e Interdependencia. Buenos Aires: Grupo Editor Latinoamericano. 1998, p. 49. 
últimos podemos identificar los tribunales de Nuremberg y Tokio como uno de los primeros antecedentes en materia de Derecho Internacional Humanitario que tras la Segunda Guerra Mundial convocó la atención de los líderes del mundo sobre la necesidad de constituir unos órganos que respaldaran a las víctimas de abusos contra la dignidad humana.

De tal forma, es posible afirmar que la Corte Penal Internacional, además de haberse constituido como una instancia supranacional garante del Derecho Internacional Humanitario, también ha llegado a posicionarse como un foro propicio para contener las acciones injustas y por demás perjudiciales emprendidas por algunos Estados que se abrogan el derecho a hacer justicia por sus propias manos. Así, se puede concluir que la existencia de este tipo de instituciones posibilita mecanismos concretos de fiscalización y judicialización de los verdaderos responsables de la degradación humana, a la cual están cada vez más pueblos expuestos.

Las reacciones a la existencia de la Corte, como un órgano que persuade de diferentes formas a los Estados e individuos para que asuman una actitud respetuosa de la dignidad humana, han sido diversas, pero en específico la asumida por Estados Unidos obstaculiza de manera evidente el avance hecho en la construcción de condiciones interdependientes que posibilitasen la desmonopolización de la gestión de asuntos de política exterior, la construcción de la Agenda Global y el uso de la fuerza como herramienta de poder.

Frente a esto es posible anotar que "...la debilidad de las organizaciones internacionales y los problemas de la obligatoriedad del derecho internacional a veces despistan a los observadores y los llevan a pensar que los regímenes internacionales son insignificantes y que pueden ignorarse por completo. Pero aunque la integración global generalizada sea débil, a menudo los regímenes internacionales tiene importantes efectos sobre las relaciones de interdependencia que involucran a unos pocos países o a muchos en un problema específico". (KEOHANE, ROBERT O., NYE, JOSEPH S. "Poder e Interdependencia". 1998).

Por consiguiente, es viable afirmar que al analizar el sistema interdependiente actual es posible identificar muestras representativas de sus principales características en diferentes ámbitos, y que en el caso de la Corte Penal Internacional genera nuevos interrogantes tales como iestá la Corte realmente preparada para un nivel mayor de interdependencia y, por lo tanto, de compromiso en el respeto y protección de la dignidad humana?, ies acaso la Corte un ejemplo representativo de la superación de las premisas realistas o simplemente las replantea en un nuevo contexto?, ¿se podría considerar la posición de Estados Unidos frente a la Corte como un fracaso de este organismo? y de ser así źqué podría aportar la Teoría de la Interdependencia para potencializar dicha situación a favor de un régimen internacional más coherente y justo con las crecientes problemáticas mundiales?

La existencia de la Corte Penal Internacional como respuesta integral a la problemática mundial de los abusos cometidos contra la dignidad humana de los individuos evidencia un interés 
profundo por convocar los esfuerzos de la Comunidad Internacional para prevenir y solucionar tales circunstancias y para sancionar a los responsables de perpetrar dichas arbitrariedades.

Todo esto visto en el marco de la Teoría de la Interdependencia, de Robert Keohane y Joseph Nye, presenta diversos matices a las tres características principales de esta Teoría, en primer lugar, la existencia de canales múltiples ejemplifica de manera apropiada todos los niveles de contacto y relacionamiento que se gestan en las diferentes dinámicas de la Corte, es decir, desde su creación las relaciones interestatales, transgubernamentales y transnacionales han forjado todo tipo de alianzas, acuerdos o decisiones conjuntas que han viabilizado los consensos respectivos en los ámbitos internos de los Estados, lo cual además se posibilita por la multiplicidad de actores que tras la globalización han entrado a formar parte del Sistema Internacional.

En segundo lugar identificamos la falta de jerarquización entre las cuestiones que componen la Agenda Global, lo cual a través del tiempo y la evolución de diversas problemáticas se ha ido alejando de las preocupaciones de orden militar (de defensa y seguridad nacional) para irse acercando a situaciones de orden económico, ambiental, cultural o social, como es el caso de los Derechos Humanos y el Derecho Internacional Humanitario, lo que en definitiva compromete a los organismos internacionales en general, y a la Corte en particular, a contribuir con la construcción democrática y pluralista de la Agenda Global asumiendo sus respectivos papeles como agentes gestores y coordinadores de los esfuerzos de la Comunidad Internacional para afrontar la diversidad de problemáticas mundiales presentes y futuras.

Finalmente, en tercer lugar se ubica el menor papel de la fuerza militar cuyas consecuencias para el equilibrio de poder, no solo en escenarios bilaterales sino además multilaterales, posibilitan que las opciones de intervenir exitosamente en los vaivenes de las relaciones internacionales sean favorables, no solo a los Estados tradicionalmente dominantes, sino también a aquellos Estados que haciendo parte del escenario internacional permanecen inmóviles ante sus problemas. Así, la Corte Penal Internacional propugna por reprimir tales situaciones por las consecuencias que estas pueden acarrear para la población mundial, debido al sometimiento al que están expuestos muchos países subdesarrollados por parte de los desarrollados, lo cual además se ve agudizado por los atropellos y abusos perpetrados por los "guardianes de la democracia" sobre pueblos que no encajan en su visión simplista pero aplastante del mundo de las relaciones internacionales.

Los autores Robert O. Keohane y Joseph S. Nye, en su libro Poder e Interdependencia, manifestaron lo siguiente, que va muy relacionado con el tema de análisis en este artículo:

"Pero en un mundo de múltiples problemas imperfectamente relacionados, en el cual las coaliciones son transnacionales y transgubernamentales, el papel potencial de las instituciones internacionales para la negociación política ha crecido enormemente. Ellas contribuyen, en particular, a establecer la agenda internacional, actúan como catalizadores para la formación de coaliciones y como escenario para iniciativas políticas y vinculación de los Estados débiles". (Keohane, R.\& Nye, J", 1998). 


\section{CONCLUSIONES}

- La soberanía de los Estados en un contexto de interdependencia como el actual no ha perdido su importancia, pues el Estado se ha visto abocado a responder ampliando y haciendo más efectivos los elementos soberanos que posee, ante la diversidad de retos que la existencia de instancias supranacionales como la Corte Penal Internacional ha traído consigo.

- La dinámica de interdependencia ha consolidado una multiplicidad de actores no estatales que comparten la escena internacional con el Estado, lo cual no le ha restado a este último el derecho soberano que posee como administrador legítimo de los intereses nacionales y como garante de estos en el contexto de instituciones supranacionales generadoras de obligaciones como lo constituye la Corte Penal Internacional.

- La justicia internacional, como ideal que soporta la existencia de la CPI, ha posibilitado una mayor dinámica en las relaciones transnacionales y, por consiguiente, continúa aportando a la construcción de un régimen internacional basado en un consenso sobre las normas, procedimientos y demás que configuran el Derecho Internacional Humanitario como esquema de conducta para los individuos a nivel mundial.

- El desarrollo de las premisas del modelo de interdependencia compleja evidenciado en la consolidación de proyectos internacionales, como lo es la Corte, responde a diversas necesidades o problemáticas globales que deben ser atendidas de la manera más diligente por parte de la "comunidad internacional", lo cual no excluye que la retórica generada por ciertos esferas de poder alrededor de dichas iniciativas refuercen prácticas de dependencia o, en su defecto, de interdependencia asimétrica.

- La Corte Penal Internacional protege la dignidad humana de las personas contra los crímenes de lesa humanidad; por esta razón, en los Estados colapsados fortalece su justicia y protege a sus conciudadanos castigando a quienes cometieron los delitos atroces en contra de ellos.

- El accionar de la Corte no tiene propósitos "imperialistas", sino que constituye un método de autoevaluación del aparato judicial de cada uno de los Estados, en materia de enjuiciamiento de responsables de delitos atroces, en pro de mantener su soberanía y discrecionalidad en materia de asuntos internos, pues lo que la CPI manifiestamente busca es que "Ios Estados modernicen sus sistemas penales, tipifiquen crímenes internacionales y fortalezcan la independencia del poder judicial, lo que tendrá un efecto positivo en la protección de los derechos humanos a escala mundial, al tiempo de asegurar la mejor cooperación entre los países y la Corte". (Zuleta J. \& Londoño, G. 2002). 


\section{BIBLIOGRAFÍA}

- $\quad$ Anellos, C. (2003). La Corte Penal Internacional. Universidad de Buenos Aires.

- $\quad$ Badie, B. (2000). Un mundo sin soberanía. Estados entre artificio y Responsabilidad. Colombia: Tercer mundo S.A.

- Camargo, P. Derecho Internacional Humanitario. Bogotá: Editorial Leyer, 2 Ed.

- Consejería Presidencial para los Derechos Humanos. (1998) Corte Penal Internacional. Colombia: Estatuto.

- Córdoba, J. (2001). .Derecho Penal Internacional. Bogotá: ediciones Jurídicas Gustavo Ibáñez.

- Córdoba, J. (2001). Estatuto de Roma de la Corte Penal Internacional.

- Fonseca, C. (2004). Elementos y Delimitación de los Crímenes Internacionales. Bogotá: editorial Nueva Jurídica.

- Greppi, E. (1999). La evolución de la responsabilidad penal individual bajo el derecho internacional. En: Comité Internacional de la Cruz Roja (Eds.) Revista Internacional de la Cruz Roja. No. 835. Ginebra: Comité Internacional de la Cruz Roja.

- Guerrero, Ó. \& Ambos K. Compiladores. (2003) El Estatuto de Roma de la Corte Penal Internacional. Bogotá: Universidad Externado de Colombia.

- Harhoff, F. (1997). Tribunal para Ruanda: algunos aspectos jurídicos En: Comité Internacional de la Cruz Roja (Eds.) Revista Internacional de la Cruz Roja. No. 144. Ginebra: Comité Internacional de la Cruz Roja.

- Herdegen, M. Derecho Internacional Público. México, D.F.: Instituto de Investigaciones Jurídicas de la UNAM.

- VILLA, J. (1995). Derecho Constitucional. General y colombiano. Bogotá: Editorial Leyer.

- Keohane, R y Nye, Joseph. (1998). Poder e Interdependencia. Buenos Aires: Grupo Editor Latinoamericano.

- Keohane, R y Nye, Joseph. (1974). Transgovermental Relations and International Organizations. World Politics, 27, No. 1.

- $\quad$ Keohane, R y Nye, Joseph. (1973). Transnational Relations and World Politics. Cambridge: Harvard UP.

- Keohane, R y Nye, Joseph. (1977). Power and Interdependence. World Politics in Transition. Boston: Little Brown and company. 
- Mesa, R. (1992). La Nueva Sociedad Internacional. Madrid: Centro de Estudios Constitucionales.

- del Arenal, C. (1993). El Nuevo Escenario Mundial y la Teoría de las Relaciones Internacionales. Madrid: Tecnos.

- Pérez, M. (2010). Organizaciones no Gubernamentales, características de otro actor en el tablero. Buenos Aires: CAEl Centro Argentino de Estudios Internacionales.

- Oficina en Colombia del Alto comisionado de las Naciones Unidas para los Derechos Humanos. (2003). Compilación de Instrumentos Internacionales, 4 Ed.

- VERGARA MOLANO ALBERTO. (2002) "Derecho internacional público" Editorial G\&B Graphic, tercera edición. Bogotá, D.C.

- Zuleta, J. \& Londoño, G. (2002). Justicia Universal en La Corte Penal Internacional. Bogotá: Librería Jurídica Sánchez R. Ltda.

- United Nations. (U.N). (n.d.). Naciones Unidas en español. Extraído febrero- abril, 2011, desde http:// www.un.org/spanish/. 